\title{
AN ALGORITHM FOR COMPUTING THE UNIVERSAL GRÖBNER BASIS OF GRAPH IDEALS
}

\author{
YANNIS C. STAMATIOU AND CHRISTOS TATAKIS
}

\begin{abstract}
The universal Gröbner basis of an ideal is a Gröbner basis with respect to all term orders simultaneously. The aim of this paper is to present an algorithmic approach to compute the universal Gröbner basis for the toric ideal corresponding to an undirected graph, based on the theoretically knowledge of this set and on a recent, efficiently computable algorithmic characterization of the Graver basis of the ideal.
\end{abstract}

\section{INTRODUCTION}

A Gröbner basis is a specific generating set of an ideal $I$ over a polynomial ring $\mathbb{K}\left[x_{1}, \ldots, x_{n}\right]$, where $\mathbb{K}$ is a field. It has extremely useful algebraic properties and it is relatively easy to extract information about the ideal, given its Gröbner basis. The study of the Gröbner bases has become a major research topic in commutative algebra, combinatorics and computer science. Gröbner basis theory provides the foundations for many algorithms in commutative algebra and algebraic geometry. Its importance stems from its wide applicability in problems coming from diverse disciplines such as mathematics, combinatorics, computer design theory, symbolic computation, integer programming, engineering, computer technology and cryptography. The concept of a Gröbner basis was introduced by Buchberger at 1965 who named this set after his supervisor W. Gröbner. Buchberger's algorithm is the most well known algorithmic method for computing a Gröbner basis for an ideal $I$ of a polynomial ring. Most of the symbolic computation software packages, such as CoCoA, Macaulay, Mathematica, Maple and Singular, include algorithms for computing this set. For more information on Gröbner bases and corresponding algorithms, see [1, 2, 3].

The universal Gröbner basis is defined as the union of all reduced Gröbner bases $G_{<}$of the ideal $I$, as $<$ runs over all term orders. It is a finite subset of $I$ and it is a Gröbner basis for the ideal with respect to all admissible term orders (for more see [9]). They were introduced by V. Weispfenning [13] and N. Schwartz [8]. There are not many classes of ideals for which we know their universal Gröbner bases. In general, characterizing and computing this set is a very difficult and computationally demanding problem. One of the known classes, is the class of the toric ideals associated to incidence matrices of graphs. The structure of the universal Gröbner basis of a toric ideal of a graph $G$, was characterized theoretically in 10, Theorem 3.4.].

The goal of this paper is to transform this theorem into an algorithm in order to compute this set. Computing the universal Gröbner basis of an ideal, is in general extremely complicated, despite the existence of a few algorithms, valuable from a

2000 Mathematics Subject Classification. Primary 14M25, 68Q40, 68W30, 13P10, 05C85. 
theoretical point of view but of a limited practical use. One would question the necessity of computing the universal Gröbner basis of the ideal, while at the same time we could just use a Gröbner basis of it under fixed term order, which would also be proper and there are already available algorithms for it. The reply stems from the fact that in certain problems one needs to compute Gröbner bases for the same toric ideal but each time with different term order. For example, problems coming from integer programming where the term order depends on a cost function that should be minimized. Then, instead of a Gröbner computation each time that cost function changes, if the universal Gröbner basis is known, we just have to compare degrees in every binomial in the universal Gröbner basis to produce a Gröbner basis for the toric ideal.

The structure of this paper is as follows. In Section 2 we present the terminology and the theory which someone needs to follow. The algorithm is presented in Section 3. Its input is the Graver basis of the ideal, which it is known algorithmically from 6. We compute the complexity of the algorithm, see Theorem 3.3. and we prove that the algorithm decides in polynomial time whether an element of the Graver basis of the ideal belongs also to its universal Gröbner basis, see Corollary 3.5.

\section{Graver basis AND the Universal GröBner BASIS OF A GRAPH IDEAL}

In this section we define the toric ideals of graphs and next we give some basic elements of graph theory which will be useful in the description of the Graver and universal Gröbner basis of a toric ideal of a graph $G$.

Let $A=\left\{\mathbf{a}_{1}, \ldots, \mathbf{a}_{m}\right\} \subseteq \mathbb{N}^{n}$ be a vector configuration in $\mathbb{Q}^{n}$ and $\mathbb{N} A:=\left\{l_{1} \mathbf{a}_{1}+\right.$ $\left.\cdots+l_{m} \mathbf{a}_{m} \mid l_{i} \in \mathbb{N}\right\}$ the corresponding affine semigroup. We grade the polynomial ring $\mathbb{K}\left[x_{1}, \ldots, x_{m}\right]$ over an arbitrary field $\mathbb{K}$ by the semigroup $\mathbb{N} A$ setting $\operatorname{deg}_{A}\left(x_{i}\right)=$ $\mathbf{a}_{i}$ for $i=1, \ldots, m$. For $\mathbf{u}=\left(u_{1}, \ldots, u_{m}\right) \in \mathbb{N}^{m}$, we define the $A$-degree of the monomial $\mathbf{x}^{\mathbf{u}}:=x_{1}^{u_{1}} \cdots x_{m}^{u_{m}}$ to be

$$
\operatorname{deg}_{A}\left(\mathbf{x}^{\mathbf{u}}\right):=u_{1} \mathbf{a}_{1}+\cdots+u_{m} \mathbf{a}_{m} \in \mathbb{N} A .
$$

The toric ideal $I_{A}$ associated to $A$ is the prime ideal generated by all the binomials $\mathbf{x}^{\mathbf{u}}-\mathbf{x}^{\mathbf{v}}$ such that $\operatorname{deg}_{A}\left(\mathbf{x}^{\mathbf{u}}\right)=\operatorname{deg}_{A}\left(\mathbf{x}^{\mathbf{v}}\right)$, see 9 . For such binomials, we set $\operatorname{deg}_{A}\left(\mathbf{x}^{\mathbf{u}}-\mathbf{x}^{\mathbf{v}}\right):=\operatorname{deg}_{A}\left(\mathbf{x}^{\mathbf{u}}\right)$. An irreducible binomial $\mathbf{x}^{\mathbf{u}}-\mathbf{x}^{\mathbf{v}}$ in $I_{A}$ is called primitive if there exists no other binomial $\mathbf{x}^{\mathbf{w}}-\mathrm{x}^{\mathbf{z}}$ in $I_{A}$ such that $\mathrm{x}^{\mathbf{w}}$ divides $\mathbf{x}^{\mathbf{u}}$ and $\mathbf{x}^{\mathbf{z}}$ divides $\mathbf{x}^{\mathbf{v}}$. The set of primitive binomials forms the Graver basis of $I_{A}$ and is denoted by $G r_{A}$.

We study a special class of toric ideals which arise from graphs. Let $G$ be a connected, undirected, finite, simple graph on the vertex set $V(G)=\left\{v_{1}, \ldots, v_{n}\right\}$. Let $E(G)=\left\{e_{1}, \ldots, e_{m}\right\}$ be the set of edges of $G$ and $\mathbb{K}\left[e_{1}, \ldots, e_{m}\right]$ the polynomial ring in the $m$ variables $e_{1}, \ldots, e_{m}$ over an arbitrary field $\mathbb{K}$. We will associate each edge $e=\left\{v_{i}, v_{j}\right\} \in E(G)$ with the element $a_{e}=v_{i}+v_{j}$ in the free abelian group $\mathbb{Z}^{n}$, where $v_{i}=(0, \ldots, 0,1,0, \ldots, 0)$ is the vector with 1 in the $i$-th coordinate. By $I_{G}$ we denote the toric ideal $I_{A_{G}}$ in $\mathbb{K}\left[e_{1}, \ldots, e_{m}\right]$, where $A_{G}=\left\{a_{e} \mid e \in E(G)\right\} \subset \mathbb{Z}^{n}$.

A walk connecting $v_{i_{1}} \in V(G)$ and $v_{i_{s+1}} \in V(G)$ is a finite sequence of the form

$$
w=\left(\left\{v_{i_{1}}, v_{i_{2}}\right\},\left\{v_{i_{2}}, v_{i_{3}}\right\}, \ldots,\left\{v_{i_{s}}, v_{i_{s+1}}\right\}\right)
$$

with each $e_{i_{j}}=\left\{v_{i_{j}}, v_{i_{j+1}}\right\} \in E(G)$. The length of the walk $w$ is the number $s$ of edges of the walk. An even (respectively odd) walk is a walk of even (respectively odd) length. A walk $w=\left(\left\{v_{i_{1}}, v_{i_{2}}\right\},\left\{v_{i_{2}}, v_{i_{3}}\right\}, \ldots,\left\{v_{i_{s}}, v_{i_{s+1}}\right\}\right)$ is called closed if 
$v_{i_{s+1}}=v_{i_{1}}$. A cycle is a closed walk

$$
\left(\left\{v_{i_{1}}, v_{i_{2}}\right\},\left\{v_{i_{2}}, v_{i_{3}}\right\}, \ldots,\left\{v_{i_{s}}, v_{i_{1}}\right\}\right)
$$

with $v_{i_{k}} \neq v_{i_{j}}$, for every $1 \leq k<j \leq s$. Note that, although the graph $G$ has no multiple edges, the same edge $e$ may appear more than once in a walk. In this case $e$ is called a multiple edge of the walk $w$. A graph is called biconnected if it is connected and does not contain a vertex whose removal increases the number of connected components of the graph. Also, we define the degree of a vertex of a graph (cor. of a walk) as the usual definition, which means as the number of the edges of the graph (cor. of the walk) incident to the vertex. Given an even closed walk of the graph $G, w=\left(e_{i_{1}}, e_{i_{2}}, \ldots, e_{i_{2 q}}\right)$ we denote by $B_{w}$ the binomial

$$
B_{w}=\prod_{k=1}^{q} e_{i_{2 k-1}}-\prod_{k=1}^{q} e_{i_{2 k}} .
$$

We remark that $B_{w} \in I_{G}$. Moreover, it is known that the toric ideal $I_{G}$ is generated by binomials of this form, see 12 . We note that the same walk can be written in different ways but the corresponding binomials may differ only in the sign. Also note that for certain even closed walks $w$ the binomial $B_{w}$ may be zero, for example take $\zeta$ to be any walk then for the even closed walk $w=(\zeta,-\zeta)$ we have $B_{w}=0$.

The relation between the Graver basis and the universal Gröbner basis, which is denoted by $\mathcal{U}_{A}$, for a toric ideal $I_{A}$ was described by B. Sturmfels:

Proposition 2.1. 9] For any toric ideal $I_{A}$ we have $\mathcal{U}_{A} \subset G r_{A}$.

Therefore the knowledge of the Graver basis for a toric ideal plays a key role for computing the $\mathcal{U}_{A}$. In order to describe the above sets in graph ideals, first, we give some extra graph notations.

We denote by w the subgraph of $G$ with vertices the vertices of the walk and edges the edges of the walk $w$. If $W$ is a subset of the vertex set $V(G)$ of $G$ then the induced subgraph of $G$ on $W$ is the subgraph of $G$ whose vertex set is $W$ and whose edge set is $\{\{v, u\} \in E(G) \mid v, u \in W\}$. The walk $w$ is primitive if and only if the binomial $B_{w}$ is primitive. A cut edge (respectively cut vertex) is an edge (respectively vertex) of the graph whose removal increases the number of connected components of the remaining subgraph. A block is a maximal connected subgraph of a given graph $G$ which does not contain a cut vertex.

The next corollary describes the elements $B_{w} \in I_{G}$ that belong to the Graver basis.

Corollary 2.2. [7, Corollary 3.3] Let $G$ be a graph and $W$ a connected subgraph of $G$. The subgraph $W$ is the graph $\mathbf{w}$ of a primitive walk $w$ if and only if

(1) $W$ is an even cycle or

(2) $W$ is not biconnected and

(a) every block of $W$ is a cycle or a cut edge and

(b) every cut vertex of $W$ belongs to exactly two blocks and separates the graph in two parts, the total number of edges of the cyclic blocks in each part is odd.

In 10 the authors gave a necessary and sufficient characterization of the universal Gröbner basis of $I_{G}$. In order to describe it, we need the notion of the pure cyclic block. 
Every even primitive walk $w=\left(e_{i_{1}}, \ldots, e_{i_{2 k}}\right)$ partitions the set of edges in the two sets $\mathbf{w}^{+}=\left\{e_{i_{j}} \mid j \quad\right.$ odd $\}, \mathbf{w}^{-}=\left\{e_{i_{j}} \mid j \quad\right.$ even $\}$, otherwise the binomial $B_{w}$ would not be irreducible. The edges of $\mathbf{w}^{+}$are called odd edges of the walk and those of $\mathbf{w}^{-}$even. Note that for an even closed walk whether an edge is even or odd depends on the edge that we start counting from. Thus, it is not important to identify whether an edge is even or odd but to separate the edges into two disjoint classes.

Definition 2.3. 10, Definition 3.1] A cyclic block $B$ of a primitive walk $w$ is called pure if all edges of $B$ are either in $\boldsymbol{w}^{+}$or in $\boldsymbol{w}^{-}$. A primitive walk $w$ is called mixed if no cyclic block of $w$ is pure.

The following Theorem describes the elements of the universal Gröbner basis of $I_{G}$, for any undirected graph $G$.

Theorem 2.4. [10, Theorem 3.4] Let $w$ be a primitive walk. $B_{w}$ belongs to the universal Gröbner basis of $I_{G}$ if and only if $w$ is mixed.

Let see an example.

Example 2.5. We consider the graph $G$ depicted in Figure 1 and let

$$
w=\left\{e_{1}, e_{2}, e_{3}, e_{4}, e_{5}, e_{6}, e_{7}, e_{8}, e_{9}, e_{10}, e_{11}, e_{12}\right\}
$$

be an even walk which we can be seen in Figure 1, Let

$$
B_{w}=\overbrace{e_{1} e_{3} e_{5} e_{7} e_{9} e_{11}}^{\mathbf{w}^{+}}-\overbrace{e_{2} e_{4} e_{6} e_{8} e_{10} e_{12}}^{\mathbf{w}^{-}}
$$

be its corresponding binomial.

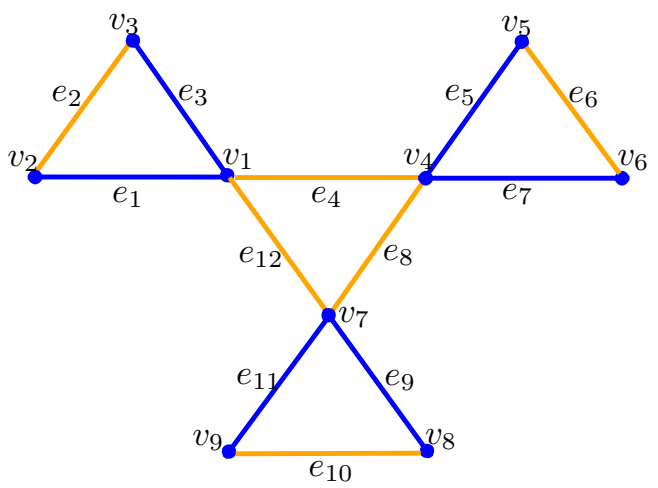

Figure 1. The graph $\mathrm{G}$

The above graph $W$ is not biconnected, each of its blocks is a cycle and every cut vertex of $W$ belongs to exactly two blocks and separates the graph in two parts, and the total number of edges of the cyclic blocks in each part is odd. From Corollary 2.2 the walk $w$ is primitive and for the corresponding binomial $B_{w}$ it holds that $B_{w} \in G r_{G}$. Also $w$ is not mixed because of the existence of the block 
$B_{1}=\left\{e_{4}, e_{8}, e_{12}\right\}$ which is not pure, since all of its edges $\left\{e_{4}, e_{8}, e_{12}\right\}$ belong to $\mathbf{w}^{-}$. Therefore from Theorem 2.4 we have that the binomial $B_{w} \notin \mathcal{U}_{G}$.

Based on Corollary 2.2 one can check that the Graver basis of the toric ideal $I_{G}$ consists of the following ten elements:

$G r_{G}=\left\{B_{1}=e_{1} e_{3} e_{8}-e_{2} e_{4} e_{12}, B_{2}=e_{4} e_{6} e_{8}-e_{5} e_{7} e_{12}, B_{3}=e_{8} e_{12} e_{10}-e_{4} e_{9} e_{11}, B_{4}=\right.$ $e_{1} e_{3} e_{5} e_{7}-e_{2} e_{4}^{2} e_{6}, B_{5}=e_{1} e_{3} e_{8}^{2} e_{6}-e_{2} e_{12}^{2} e_{7} e_{5}, B_{6}=e_{1} e_{3} e_{11} e_{9}-e_{2} e_{12}^{2} e_{10}, B_{7}=$ $e_{1} e_{3} e_{8}^{2} e_{10}-e_{2} e_{4}^{2} e_{9} e_{11}, B_{8}=e_{11} e_{9} e_{4}^{2} e_{6}-e_{10} e_{12}^{2} e_{5} e_{7}, B_{9}=e_{11} e_{9} e_{7} e_{5}-e_{10} e_{8}^{2} e_{6}, B_{10}=$ $\left.B_{w}=e_{1} e_{3} e_{5} e_{7} e_{9} e_{11}-e_{2} e_{4} e_{6} e_{8} e_{10} e_{12}\right\}$.

\section{Algorithmic description of the Universal Gröbner Basis of $I_{G}$}

In this section we state the main result of this paper which gives an algorithmic description of the universal Gröbner basis of a toric ideal of a graph $G$. We remind that $A=\left\{\mathbf{a}_{1}, \ldots, \mathbf{a}_{m}\right\} \subseteq \mathbb{N}^{n}$ is a vector configuration in $\mathbb{Q}^{n}$. For the algorithm that we will present, its input is the set of the primitive elements of a toric ideal of a graph $G$, i.e. its Graver basis. There are a lot of algorithms which give the Graver basis of an ideal. In [9, Sturmfels gave algorithms for computing a Graver basis by Lawrence lifting of $A$ and the universal Gröbner basis for toric ideals with an algebraic geometry view. Moreover, he gave an algorithm, which takes as input the universal Gröbner basis of $I_{A}$ in order to produce the State polytope of the ideal, see [9, Theorem 7.15]. In 6] M. Ogawa, H. Hara and A. Takemura gave an algorithm for sampling elements from the Graver basis set of $I_{G}$, which was associated with a simple undirected graph for testing the beta model of graphs by Markov chains, based on Monte Carlo methods. In general, the Graver basis of toric ideals of graphs can be computed by symbolic computation software packages such as the 4ti2 (see [11]) or Macaulay 2 (see [5]).

The next corollary is useful for the correctness of our algorithm and it follows from Theorem 2.4

Corollary 3.1. Let $w$ be a primitive walk of a graph $G$. Then for the binomial $B_{w}$ it holds $B_{w} \in \mathcal{U}_{G}$ if and only if the set of its odd edges and the set of its even edges do not contain a cycle of $w$.

Proof. Let $w$ be a primitive walk of a graph $G$. Since $w$ is primitive, from Corollary 2.2 it follows that all of its blocks are either cycles either cut edges. From Theorem 2.4, $B_{w} \in \mathcal{U}_{G}$ if and only if $w$ is mixed. By definition, $w$ is mixed if and only if all of its cyclic blocks are not pure, i.e. for every block $B_{i}$ of its blocks, $E\left(B_{i}\right) \nsubseteq E\left(\mathbf{w}^{+}\right)$and $E\left(B_{i}\right) \nsubseteq E\left(\mathbf{w}^{-}\right)$, where by $E\left(B_{i}\right), E\left(\mathbf{w}^{+}\right), E\left(\mathbf{w}^{-}\right)$we denote the sets of edges of the corresponding graphs.

From Theorem 2.4. we are interested only in the mixed walks $w$ of the graph, which means that we are interested only in the cyclic blocks of the walk $w$. The result now follows.

Let see an example.

Example 3.2. We stay on Example 2.5 and we consider the walk

$$
w=\left\{e_{1}, e_{2}, e_{3}, e_{4}, e_{5}, e_{6}, e_{7}, e_{8}, e_{9}, e_{10}, e_{11}, e_{12}\right\},
$$

where $B_{w}=\overbrace{e_{1} e_{3} e_{5} e_{7} e_{9} e_{11}}^{\mathbf{w}^{+}}-\overbrace{e_{2} e_{4} e_{6} e_{8} e_{10} e_{12}}^{\mathbf{w}^{-}}$is its corresponding binomial. We note that the cycles of the walk $w$ are:

$$
c_{1}=\left(e_{1}, e_{2}, e_{3}\right), c_{2}=\left(e_{4}, e_{8}, e_{12}\right), c_{3}=\left(e_{5}, e_{6}, e_{7}\right), c_{4}=\left(e_{9}, e_{10}, e_{11}\right)
$$


We remark that there is the cycle $c_{2}$ such that its edges are all even. By the previous corollary it follows that $B_{w} \notin \mathcal{U}_{G}$.

Now we are ready to describe our algorithm. Its correctness is guaranteed by Corollary 3.1. The algorithm takes as input the elements $B_{w} \in G r_{G}$ as graphs $\left(V\left(B_{w}\right), E\left(B_{w}\right)\right)$ separated into its two components, $\mathbf{w}^{+}$and $\mathbf{w}^{-}$in their graph representation (as, not-induced, subgraphs of $G),\left(V\left(\mathbf{w}^{+}\right), E\left(\mathbf{w}^{+}\right)\right)$and $\left(V\left(\mathbf{w}^{-}\right), E\left(\mathbf{w}^{-}\right)\right)$ respectively (i.e. the vertices and edges of each subgraph). We remark that the graph representation is not a multigraph, i.e. edges appear only once regardless of the number of times they appear in $B_{w}$. At each iteration, the algorithm considers one by one the elements $B_{w} \in G r_{G}$ using their graph representation. For each $B_{w}$, the goal of the algorithm is to check whether there is a cycle either in $\left(V\left(\mathbf{w}^{+}\right), E\left(\mathbf{w}^{+}\right)\right)$either in $\left(V\left(\mathbf{w}^{-}\right), E\left(\mathbf{w}^{-}\right)\right)$. According to Corollary 3.1, if such a cycle exists then $B_{w} \notin \mathcal{U}_{G}$, otherwise $B_{w} \in \mathcal{U}_{G}$.

More specifically, by Corollary 2.2, the vertices of the graphs $\left(V\left(\mathbf{w}^{+}\right), E\left(\mathbf{w}^{+}\right)\right)$ and $\left(V\left(\mathbf{w}^{-}\right), E\left(\mathbf{w}^{-}\right)\right)$have degree either 1 either 2 . The algorithm, thus, attempts to build a cycle first in $\left(V\left(\mathbf{w}^{+}\right), E\left(\mathbf{w}^{+}\right)\right)$and then in $\left(V\left(\mathbf{w}^{-}\right), E\left(\mathbf{w}^{-}\right)\right)$by choosing as starting points vertices of degree 2 , if such vertices exist. If such a cycle is found then, by Corollary $3.1, B_{w} \notin \mathcal{U}_{G}$, otherwise $B_{w} \in \mathcal{U}_{G}$.

The formal description of this algorithm, in pseudocode, follows below.

\section{ALGORITHM UGB}

\section{INPUT:}

The elements $B_{w} \in G r_{G}$, each split into its two parts, i.e. $B_{w}^{+}$and $B_{w}^{-}$, given as simple (i.e. edges have multiplicity 1 ) subgraphs of $G$ $\left(V\left(\mathbf{w}^{+}\right), E\left(\mathbf{w}^{+}\right)\right)$and $\left(V\left(\mathbf{w}^{-}\right), E\left(\mathbf{w}^{-}\right)\right)$respectively.

OUTPUT: the elements of the universal Gröbner basis $\mathcal{U}_{G}$.

\section{LOCAL VARIABLES:}

Visited: $1 \times n$ array of boolean false that is used in the cycle finding process. stop: boolean variable.

$i, j$ : integers.

$U$ : a set of graph edges (i.e. non-ordered pairs of vertices).

\section{1. begin algorithm}

2. $\mathcal{U}_{G} \leftarrow \emptyset$

3. for all elements $B_{w} \in G r_{G}$

4. $\quad$ stop $\leftarrow$ false

5. for $s \in\{+,-\}$

6.

7.

8.

9.

10.

11.

12.

13.

14.

15.

16.

17.

18.

19.

20.

21.
Initialize Visited to false

while $\exists i \in V\left(\mathbf{w}^{s}\right)$ such that $\operatorname{deg}\left(v_{i}\right)=2$ and $\operatorname{Visited}[i]=$ false and stop = false

Visited $[i] \leftarrow$ true.

$U \leftarrow \emptyset$

repeat

Select $j$, such that $\left\{v_{i}, v_{j}\right\} \notin U$ and $\left\{v_{i}, v_{j}\right\} \in E\left(\mathbf{w}^{s}\right)$

$U \leftarrow U \cup\left\{v_{i}, v_{j}\right\}$

if Visited $[j]=$ true

stop $\leftarrow$ true

until stop $=$ true or $\operatorname{deg}\left(v_{j}\right)=1$

if $\operatorname{deg}\left(v_{j}\right)=1 / /$ No cycle was encountered during this iteration.

$E\left(\mathbf{w}^{s}\right) \leftarrow E\left(\mathbf{w}^{s}\right)-U / /$ Delete encountered edges, updating vertices' degrees.

end while // "While" at line 7 .

end for // "For" at line 5.

if stop $=$ false

$\mathcal{U}_{G} \leftarrow \mathcal{U}_{G} \cup B_{w}$ 
22. end for // "For" at line 3.

23. return $\mathcal{U}_{G}$

24. end algorithm

In the next theorem we prove the correctness and determine computational complexity of the algorithm UGB and show that in order to decide algorithmically whether a single element of the Graver basis of $I_{G}$ belongs also to $\mathcal{U}_{G}$ it requires polynomial, in the number of the vertices of $G$, computational steps. By $\left|G r_{G}\right|$ we denote the number of the elements of the Graver basis of $I_{G}$ and by $\left|B_{w}\right|$ the number of the edges in the walk $w$.

Theorem 3.3. The Algorithm UGB correctly computes the set $\mathcal{U}_{G}$ for a toric ideal of a given graph $G$ and its time complexity (number of graph edges followed by the algorithm) is

$$
\left|G r_{G}\right| \cdot \max _{w}\left(O\left(\left|B_{w}\right|\right)\right) .
$$

Proof. The algorithm is based on Corollary 3.1. Accordingly, the loop at lines 3 to 22 iterates over all elements $B_{w}$ in the Graver basis of the ideal. For each such element $B_{w}$ the algorithm examines, in turn, $B_{w}^{+}$and $B_{w}^{-}$in the loop at lines 5 to 19 , for existence of cycles within either of these two sets. The cycle finding process is in the loop at lines 7 to 18 . The "stop" variable is used in order to stop the iterations when a cycle is found (it takes the value "true" and stops the iterations at lines 7 to 18$)$.

The loop at lines 7 to 18 selects, at each iteration, an, yet, unvisited vertex of degree two, if it exists, and attempts to build a cycle starting from it. The cycle building part is composed of the loop at lines 10 to 15 . The loop, repeatedly, selects consecutive edges until either a cycle is found either a vertex of degree one is encountered. Note that according to Corollary 2.2, the vertices of the graphs that correspond to the elements $B_{w}$ of the Graver set have degree either one either two.

The "if" statement at lines 16 checks whether the loop at lines 10 to 15 was terminated due to finding a vertex of degree one, in which case no cycle was detected. In this case, the encountered edges in the set $U$ are discarded and the cycle detection process starts, again, at line 7 .

After the loop at lines 5 to 19 terminates, the "if" statement checks whether a cycle was detected in either $B_{w}^{+}$or $B_{w}^{-}$. This is the case where the variable "stop" has remained to its initial value false. Thus, the element of the Graver basis that was examined is inserted into $\mathcal{U}_{G}$ otherwise the element is discarded. Then the algorithm starts over the iteration at lines 3 to 22 to examine the next element of the Graver basis. According to Corollary 3.1 the algorithm correctly computes the elements of the Gröbner basis.

With respect to time complexity, there are $\left|G r_{G}\right|$ iterations of the loop at lines 3 to 22 . For each such iteration, the graph formed by $B_{w}$ is examined at lines 5 to 19 , separated into the components $B_{w}^{+}$and $B_{w}^{-}$in subgraph representation. Then the loop at lines 7 to 18 iteratively selects a vertex of degree 2 in order to follow consecutive vertices, at the loop at lines 10 to 15, towards detecting a cycle, if it exists. Each vertex is considered once by the loop. Then the loop at lines 10 to 15 follows consecutive edges in order to detect a cycle or reach a vertex of degree 1. Again, the encountered edges are considered once by the loop and once more, at most, after the loop, at line 17 . Thus, in total, the complexity of the algorithm 
for a specific $B_{w}$ is in the order of $\left|V\left(B_{w}\right)\right|+\left|E\left(B_{w}\right)\right|$ or (in complexity theoretic notation) $O\left(\left|V\left(B_{w}\right)\right|+\left|E\left(B_{w}\right)\right|\right)$. In our convention, the number of edges of the graph corresponding to $B_{w}$ is the number of its edges, denoted by $\left|B_{w}\right|$. Thus, the complexity for $B_{w}$ is $O\left(\left|V\left(B_{w}\right)\right|+\left|B_{w}\right|\right)$. As the number of vertices in a connected graph cannot be more than its edges minus 1 , the complexity is $O\left(\left|B_{w}\right|\right)$. Thus, in the worst case, the complexity of the algorithm for locating all elements $B_{w}$ in the Graver basis $G r_{G}$ that also belong to the Gröbner basis is $\left|G r_{G}\right| \cdot \max _{w}\left(O\left(\left|B_{w}\right|\right)\right)$.

In [10] an upper bound for the degrees of the primitive elements of $I_{G}$ is described. This bound is described in the next proposition. It will be useful in estimating the computational complexity of the algorithm.

Proposition 3.4. 10, Corollary 4.2.] Let $G$ be a graph with $n$ vertices, $n \geq 4$. The largest degree d of any binomial in the Graver basis (and in the universal Gröbner basis) for $I_{G}$ is $d \leq n-2$.

From the above proposition we know that $\left|B_{w}\right| \leq 2(n-2)$. Therefore, the following corollary holds, with $O(f(n))$ denoting functions of $n$ bounded above by $c f(n)$, for some constant $c>0$ and all $n>n_{0}$, for some constant $n_{0}$.

Corollary 3.5. The time complexity of Algorithm $U G B$ is $O\left(\left|G r_{G}\right| \cdot n\right)$.

Let us consider two examples of the operation of the algorithm based on Example 2.5 .

Example 3.6. We first consider the operation of the algorithm on the element $B_{1} \in G r_{G}$ that appears in Figure 1 with $B_{1}^{+}$in blue color and $B_{1}^{-}$in orange. The algorithm enters the loop at line 7 trying to identify an unvisited vertex of degree two. We assume that it selects $v_{1}$. Then it starts selecting consecutive vertices at the loop at line 10 . There is only one such vertex, $v_{3}$, along edge $e_{3}$, which is followed by the algorithm. Since the degree of $v_{3}$ is one, the algorithm stops and tries, at line 7 , to select another vertex of degree two, after discarding edge $e_{3}$. The only choices are $v_{4}$ or $v_{7}$. The algorithm works as with vertex $v_{1}$ concluding that in $B_{1}^{+}$there is no cycle. Then the loop at line 5 proceeds with $B_{1}^{-}$, i.e. the orange edges. There are two choices of vertices of degree two. Let us assume that at line $7 v_{1}$ is chosen. Then it is clear that the loop at line 10 will follow the vertices, detecting the cycle and rejecting $B_{1}$.

Example 3.7. Let us, now, turn to the example in Figure 2 with the algorithm first considering the blue edges, i.e. $B_{5}^{+}$.

Again, the algorithm selects a vertex of degree two trying to locate a cycle, e.g. vertex $v_{1}$. Then, the algorithm selects the next unvisited vertex, say $v_{3}$. At this point, it has reached a vertex of degree one and, thus, it stops without detecting a cycle. Then it removes the edge it followed to repeat the process. However, now at line 7 the algorithm does not find any vertex with degree two, proceeding with $B_{5}^{-}$(orange edges) at line 5 . Now there is only one vertex of degree two, $v_{4}$, which the algorithm selects. Let us assume that it, then, selects $v_{5}$. This vertex has degree one and, thus, the algorithm deletes the vertex and returns to line 7 in order to select another vertex of degree two. However, such a vertex does not exist and, thus, the algorithm is directed to line 21 , without having detected a cycle. Therefore, it includes the element $B_{5}$ in $\mathcal{U}_{G}$. 


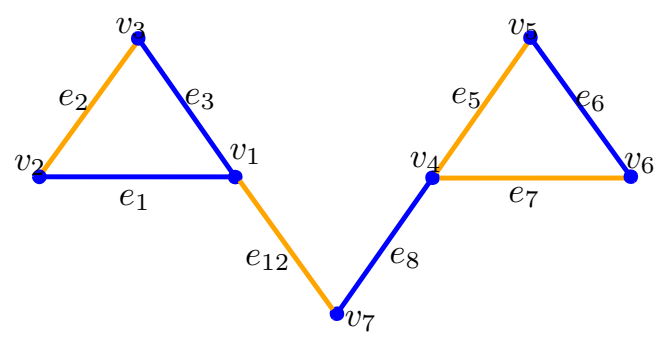

Figure 2. The binomial $B_{5}=e_{1} e_{3} e_{8}^{2} e_{6}-e_{2} e_{12}^{2} e_{7} e_{5}$

\section{Conclusion}

We provide a new algorithm for computing the universal Gröbner basis of a toric ideal associated to incidence matrices of graphs.

As it is evident from the proof of Theorem 3.3 the algorithm UGB decides in polynomial time whether a single element of the Graver basis of $I_{G}$ belongs also to $\mathcal{U}_{G}$. However, since the algorithm must test exhaustively all elements of the Graver basis for inclusion in the universal Gröbner basis, the factor $\left|G r_{G}\right|$ is present in the final complexity figure. For some classes of graphs this factor is not prohibitively large, i.e. it is polynomial in $n$. For instance, the class of graphs that consists of even cycles connected by paths where each cut vertex belongs to exactly two blocks has at most $\frac{n}{4}$ cycles since every cycle has at least four edges. Therefore its Graver basis has cardinality $\left|G r_{G}\right| \leq \frac{n}{4}$. For such graphs, the complexity of the algorithm UGB is (from Corollary 3.5) $O\left(n^{2}\right)$ which is a polynomial in the number $n$ of vertices of the graph.

However, in many cases the size of the Graver basis appears to grow exponentially fast with $n$. Thus, due to its large size, in general, there can be no polynomial time algorithm for computing the elements of the Graver basis of a general toric ideal. As an indication of the computational difficulty of this problem, in [4] J. De Loera, B. Sturmfels and R. Thomas showed, computationally, that the number of the primitive elements of $I_{K_{8}}$ is 45570 , where $K_{8}$ is the complete graph on eight vertices.

In general, there are no (to the best of our knowledge) general tight upper bounds to the cardinality of the Graver basis of a graph since this cardinality depends on its structure. Only a rough idea for the size of the Graver basis of a toric ideal of a graph $G$ can be obtained from the bound to the degrees of its elements (see Proposition 3.4). However, the actual size of the Graver basis itself can be huge.

For toric ideals of graphs, the problem of computing algorithmically the Graver basis reduces to finding the set of even closed walks of a corresponding graph $G$, which is again a very large set. We note that for the even closed walks which we are interested in, by Proposition 3.4 we pass from each edge at most two times which means that the set of the even closed walks which we are looking for is finite. To the best of our knowledge, existing algorithmic techniques can only sample elements of the Graver basis in polynomial time (see [6]) or compute Graver basis by specialized mathematical software, such as 4ti2 (see [11]), for toric ideals of graphs with small Graver basis. 
YANNIS C. STAMATIOU AND CHRISTOS TATAKIS

\section{REFERENCES}

[1] W. Adams, P. Loustaunau, An Introduction to Gröbner Bases, Graduate Studies in Mathematics, Vol. 3 AMS, (1994).

[2] T. Becker, H. Kredel, V. Weispfenning, Gröbner Bases: A Computational Approach to Commutative Algebra, Graduate Texts in Mathematics, Springer, New York, (1993).

[3] D. Cox, J. Little, D. O'Shea, Ideals, Varieties and Algorithms, Undergraduate Texts in Mathematics, Springer, New York, (1992).

[4] J. De Loera, B. Sturmfels and R. Thomas, Gröbner bases and triangulations of the second hypersimplex, Combinatorica 15 (3) (1995) 409-424.

[5] Grayson, D. and Stillman, M., Macaulay 2, a software system for research in algebraic geometry, 19932008, available from http://www.math.uiuc.edu/Macaulay2

[6] M. Ogawa, H. Hara, A. Takemura, Graver basis for an undirected graph and its application to testing the beta model of random graphs, Ann. Inst. Stat. Math. 65 (1) (2013) 191-212.

[7] E. Reyes, Ch. Tatakis, A. Thoma, Minimal generators of toric ideals of graphs, Advances in Appl. Math. 48(1) (2012) 64-78.

[8] N. Schwartz, Stability of Gröbner Bases, J. Pure Appl. Algebra 53 (1988) 171-186.

[9] B. Sturmfels, Gröbner Bases and Convex Polytopes. University Lecture Series, No. 8 American Mathematical Society Providence, R.I. (1995).

[10] Ch. Tatakis, A. Thoma, On the universal Gröbner bases of toric ideals of graphs, J. Combin. Theory Ser. A 118 (2011) 1540-1548.

[11] 4ti2 team (2008). 4ti2, a software package for algebraic, geometric and combinatorial problems on linear spaces. Available at http://www.4ti2.de

[12] R. Villarreal, Rees algebras of edge ideals, Comm. Algebra 23 (1995) 3513-3524.

[13] V. Weispfenning, Constructing universal Gröbner Bases, in Proceedings AAEEC 5, Menorca, Springer Lecture Notes in Computer Science 356 (1987) 408-417.

Dept. of Business Administration, University of Patras and Computer Technology Institute and Press - "Diophantus", Patra, Greece

E-mail address: stamatiu@ceid.upatras.gr

Department of Mathematics, University of Ioannina, Ioannina 45110, Greece

E-mail address: chtataki@cc.uoi.gr 